\title{
Controlling of the Environmental Effects of Air Shock Wave Due to Blasting In Quarry Mines
}

\author{
Zaw Moon Aung ${ }^{1}$ Myo Min Lwin ${ }^{2}$ \\ Demonstrator $^{1}$ and Professor ${ }^{2}$ \\ Department of Mining Engineering \\ Mandalay Technological University, Mandalay Division, Mandalay \\ Myanmar
}

\begin{abstract}
This paper presents controlling of the environmental effects of air shock wave due to blasting in quarry mines. The aim of this study is to predict the air overpressure due to blasting, in order to protect the dwelling area adjacent to the quarry. Prediction of air overpressure is important for mine operators who utilize blasting to ensure their blasts will not cause damage to nearby structures and stay within regulatory limits. Airblast is dependent on the size of the blast and air shock wave magnitude is dependent on how far away from the blast a recording device is located. Air shock waves are an integral part of the process of rock blasting and consequently they are unavoidable. In this paper, important and widely used empirical formulae have been used to predict the air over pressure for quarry mines. Moreover, a comparative analysis between the results obtained for constant charge per delay of $100 \mathrm{~kg}$, and monitoring distance of $20 \mathrm{~m}, 30 \mathrm{~m}$ and $40 \mathrm{~m}$ were carried out to select the suitable empirical formula. The paper concludes with the guidelines for applying the air overpressure wave based on the various distances and amount of charges per delay.
\end{abstract}

Key Words: Controlling, Air Shock Wave, Quarry Mines, Predict, Empirical Formulae, Comparative Analysis.

\section{INTRODUCTION}

Blasting is widely used in quarry mines. When explosive charges detonate in rock, most of the energy is used in breaking and displacing the rockmass. However, some of energy is released in the form of ground vibration and air-overpressure.

Blasting operations may cause excessive environmental impacts such as ground vibration, air shock wave, dust, and flyrock. Because blasting operations often focus on controlling fragmentation while neglecting the environmental consequences. Air shock wave is one of the undesirable effects and that occurs with the blasting. Air shock wave resulting from blasting can cause damage and nuisance to nearby civilians. Thus, it is important to be able to predict air shock wave accurately.

Air shock wave is a pressure wave and is also known as air vibrations, air overpressure or airborne shockwave. Air shock wave effects will always accompany blasting of rock, and will never be eliminated. The most important parameters to estimate the level of air shock wave are the amount of charge per delay and the distance to the measuring station. Moreover, the stemming length is also an important parameter.

In this study, empirical formulas and excel program are used to predict the level of air shock wave for quarry mines. The air shock wave damage and annoyance may be influenced by numerous factors such as blast design, weather, field characteristics, and human response.

\section{METHODOLOGY}

In order to meet the study objectives, firstly, Literature reviews were carried out to estimate the level of air over pressure. Secondly, important and widely used empirical formulae analysis were carried out to select the optimum empirical equation by using the excel program. Finally, predictions of air overpressure level were carried out with the various distances and amount of explosive (charge per delay) to control the blasting impact (air over pressure).

Several empirical predictors have been proposed by various scholars to estimate air shock wave level, but these methods are inapplicable in many conditions. In this paper, the following empirical formulas are used to predict the air shock wave level for quarry mines. Table 1 shows different empirical equations and site constants. 
International Journal of Advances in Scientific Research and Engineering (ijasre), Vol 5 (10), October-2019

Table 1. Different empirical equations and site constants

\begin{tabular}{|c|c|c|c|}
\hline References & Equations & $\mathbf{H}$ & B \\
\hline Siskind et al. (1980) & $\mathrm{AOp}=\mathrm{H}\left(\mathrm{DW}^{-0.33}\right)^{-\mathrm{B}}$ & 622 & 0.515 \\
\hline United States Bureau of Mines & $\mathrm{AOp}=\mathrm{H}\left(\mathrm{DW}^{-0.33}\right)^{-\mathrm{B}}$ & 1.32 & 0.97 \\
\hline USBM RI 8485 (1980) & & & \\
\hline $\begin{array}{l}\text { National Association of Australian State Road } \\
\text { Authorities, NAASRA (1983) }\end{array}$ & $\mathrm{AOp}=\left(140 \times(\mathrm{W} / 200)^{0.33}\right) / \mathrm{D}$ & - & - \\
\hline Hajihassani et al. & $\mathrm{AOp}=\mathrm{H}\left(\mathrm{DW}^{-0.33}\right)^{-\mathrm{B}}$ & $10,909.000$ & 1.09 \\
\hline Atlas Powder (1987) & $\mathrm{AOp}=\mathrm{H}\left(\mathrm{W}^{0.33} / \mathrm{D}\right)^{-\mathrm{B}}$ & 3.3 & 1.2 \\
\hline Persson et al. (1994) & $\mathrm{AOp}=\mathrm{H}\left(\mathrm{W}^{0.33} / \mathrm{D}\right)$ & 0.7 & 1 \\
\hline Kuzu et al. (2009) & $\mathrm{AOp}=\mathrm{H}\left(\mathrm{DW}^{-0.33}\right)^{-\mathrm{B}}$ & 261.54 & 0.706 \\
\hline
\end{tabular}

Where, AOp is air overpressure, $\mathrm{H}$ and B are site factors. D is the distance ( $\mathrm{m}$ or $\mathrm{ft}), \mathrm{W}$ is the charge weight per delay $(\mathrm{kg}$ or lb).

\section{THE EFFECTS OF AIR OVERPRESSURE DUE TO BLASTING}

The relationship of decibels to pressure and probable result of various airblast intensities are presented in table 2 . The equivalent wind gust velocities are also given for several intensities.

Table 2. Result of various airblast intensities [3]

\begin{tabular}{|c|c|c|c|}
\hline \multicolumn{2}{|c|}{ Airblast Intensity } & \multirow[t]{2}{*}{ Probable Result } & \multirow[t]{2}{*}{ Average Human Response } \\
\hline dB & Psi & & \\
\hline 180 & 2.900 & Structural damage & Ear drum rupture possible \\
\hline 175 & 1.631 & & \\
\hline 170 & 0.917 & Many windows break & Intolerable \\
\hline 165 & 0.516 & & \\
\hline 160 & 0.290 & & \\
\hline 155 & 0.163 & Equal to a $96 \mathrm{mph}$ wind gust & \\
\hline 150 & 0.092 & Poorly mounted windows can break & \\
\hline 145 & 0.052 & & \\
\hline 140 & 0.029 & Equal to a $40 \mathrm{mph}$ wind gust & Distinctly unpleasant \\
\hline 135 & 0.0145 & OSMRE and USBM limit & \\
\hline 130 & 0.0092 & Equal to a $23 \mathrm{mph}$ wind gust & \\
\hline 125 & 0.0052 & & \\
\hline 120 & 0.0029 & & Mildly unpleasant \\
\hline 115 & 0.0016 & & \\
\hline 110 & 0.00092 & & \\
\hline 105 & 0.00052 & & \\
\hline 100 & 0.00029 & Equal to a $7.2 \mathrm{mph}$ wind gust & \\
\hline 95 & 0.00016 & & \\
\hline 90 & 0.000092 & & Strongly Perceptible \\
\hline 85 & 0.000052 & & \\
\hline 80 & 0.000029 & & \\
\hline 75 & 0.000016 & & \\
\hline 70 & 0.0000092 & & Distinctly Perceptible \\
\hline 65 & 0.0000052 & & \\
\hline 60 & 0.0000029 & & Perceptible \\
\hline
\end{tabular}


International Journal of Advances in Scientific Research and Engineering (ijasre), Vol 5 (10), October-2019

\section{ANALYSIS OF EMPIRICAL FORMULA}

In this paper, a comparative analysis between the results obtained with constant charge per delay of $100 \mathrm{~kg}$ and monitoring distance of $20 \mathrm{~m}, 30 \mathrm{~m}$ and $40 \mathrm{~m}$ were carried out by using the various empirical formulas and excel program to select the suitable empirical formula for quarry mines. Figures 1 to 7 show the results of air overpressure level of different formulas.

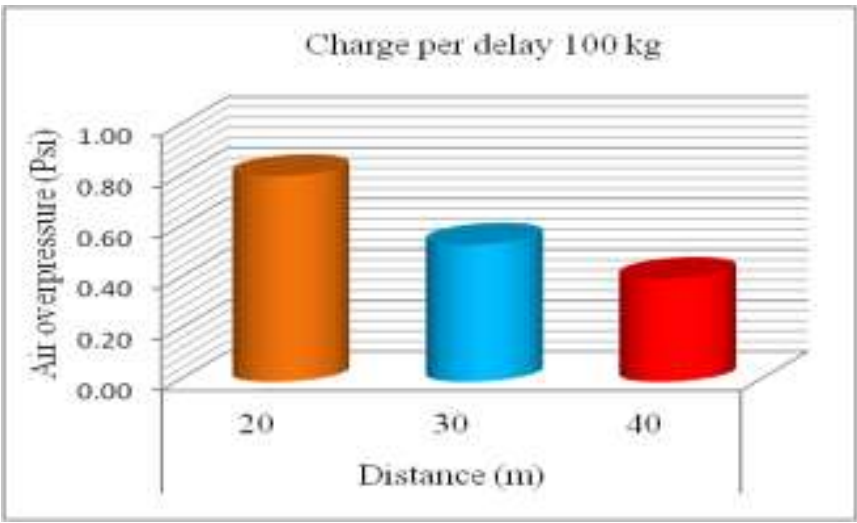

Figure 1. Air overpressure level of Siskind et al.

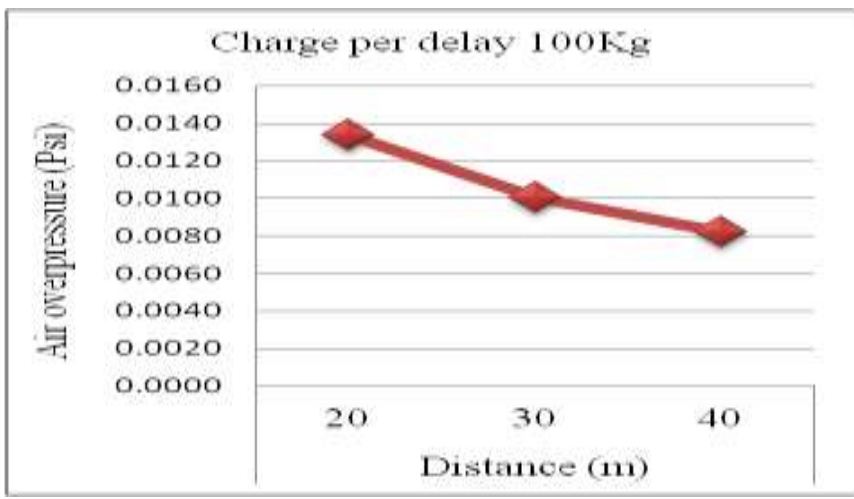

Figure 3. Air overpressure level of NAASRA method

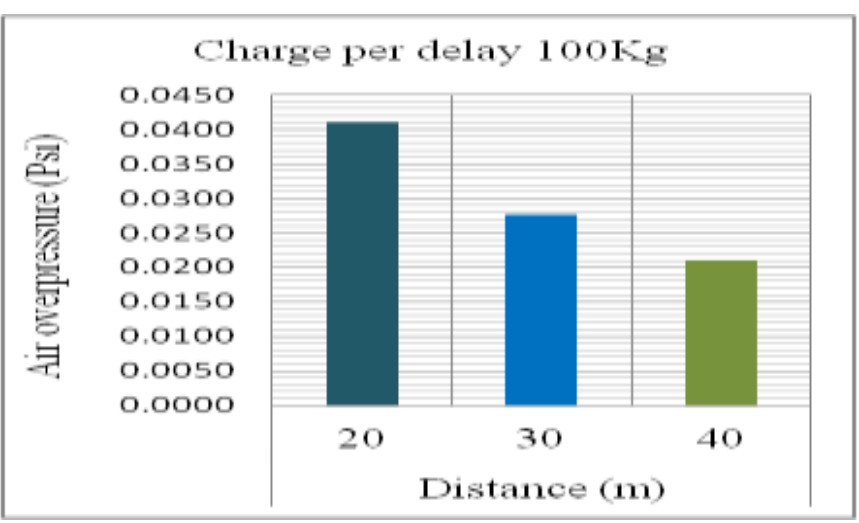

Figure 5. Air overpressure level of Atlas Powder

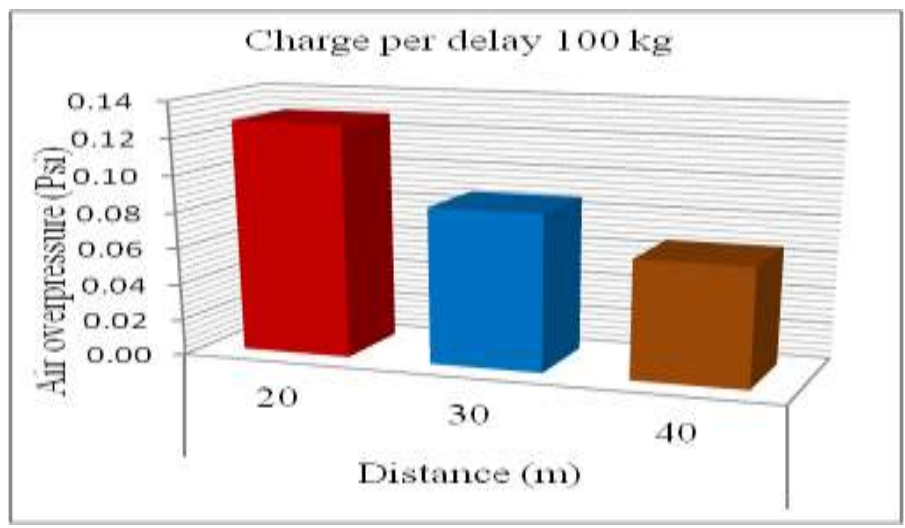

Figure 2. Air overpressure level of USBM method

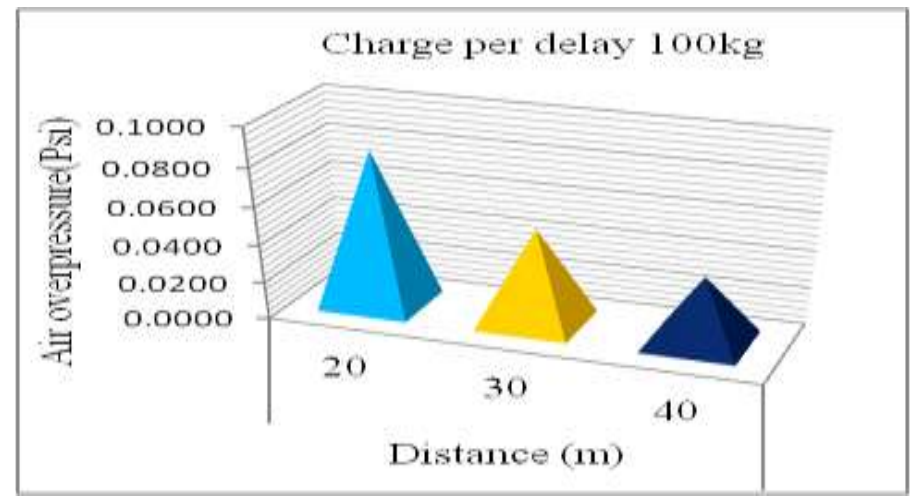

Figure 4. Air overpressure level of Hajihassani et al.

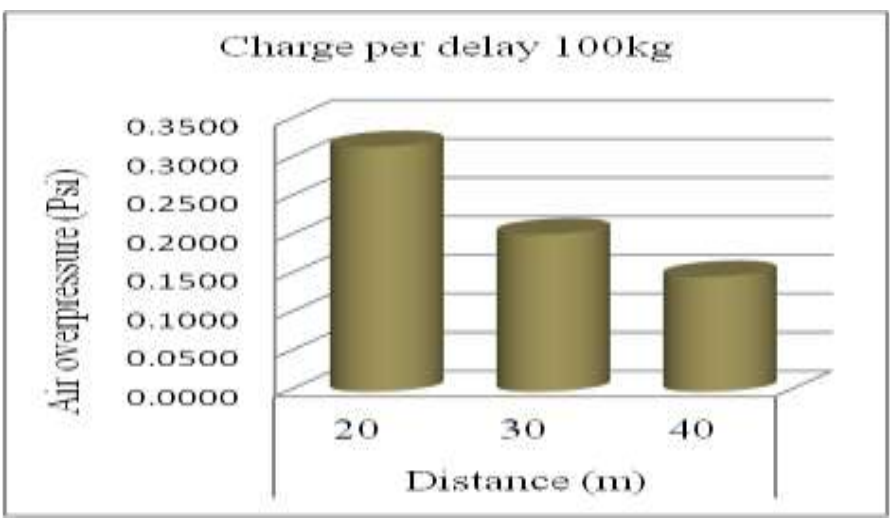

Figure 6. Air overpressure level of Persson et al.

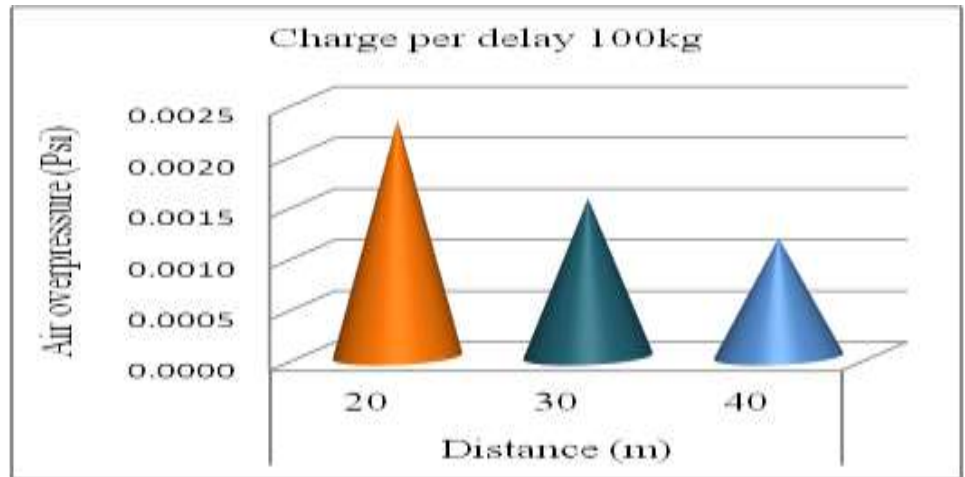

Figure 7. Air overpressure level of Kuzu et al. 
Figures 8 to 10 show the results of air overpressure for charge per delay $100 \mathrm{~kg}$ and distance $20 \mathrm{~m}$ with the various empirical formulas. According to the figure 8, 9 and 10 results, National Association of Australian State Road Authorities, NAASRA (1983) method is the highest air overpressure level. Persson et al. (1994) method is the lowest air overpressure level. In this paper, the highest air overpressure (NAASRA method) is used to predict the maximum peak particle velocity for safety condition.

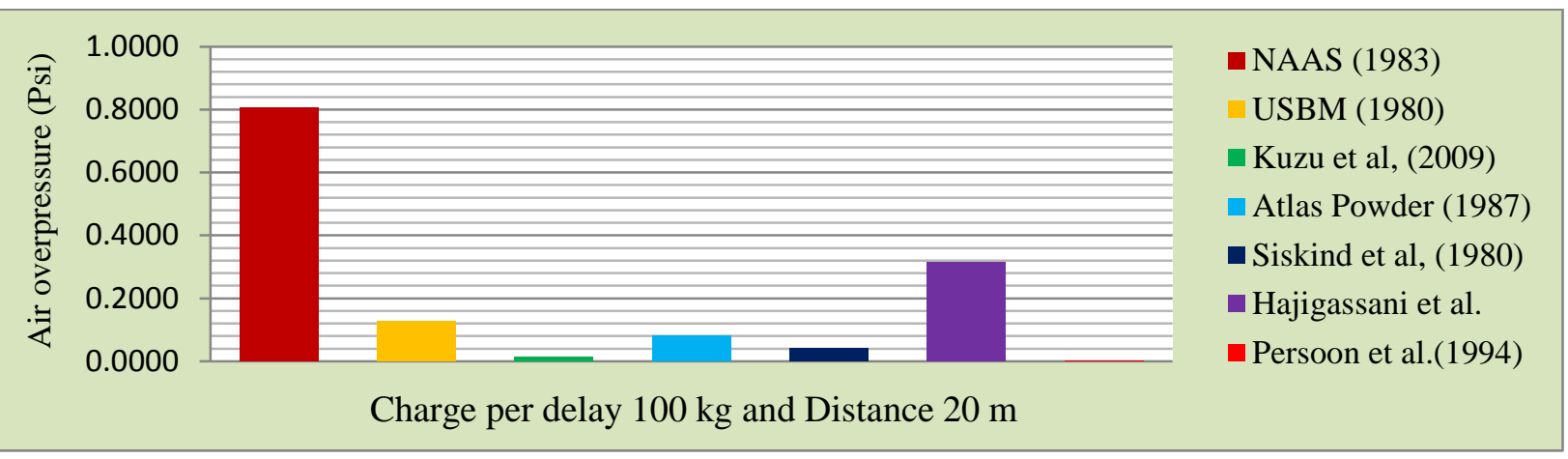

Figure 8. The results of air overpressure for charge per delay $100 \mathrm{~kg}$ and distance $20 \mathrm{~m}$

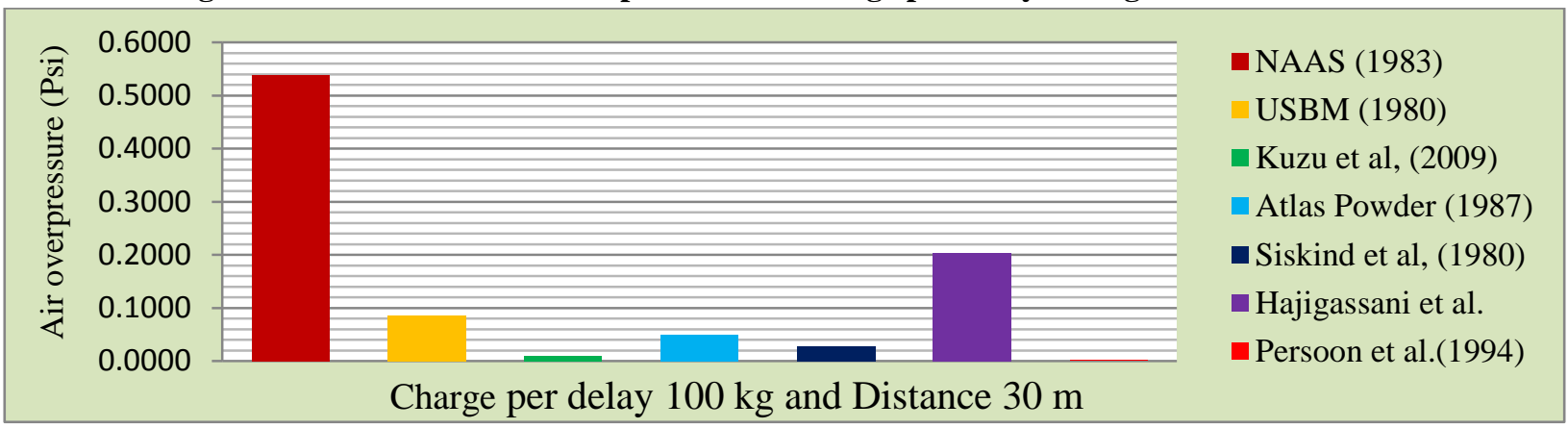

Figure 9. The results of air overpressure for charge per delay $100 \mathrm{~kg}$ and distance $30 \mathrm{~m}$

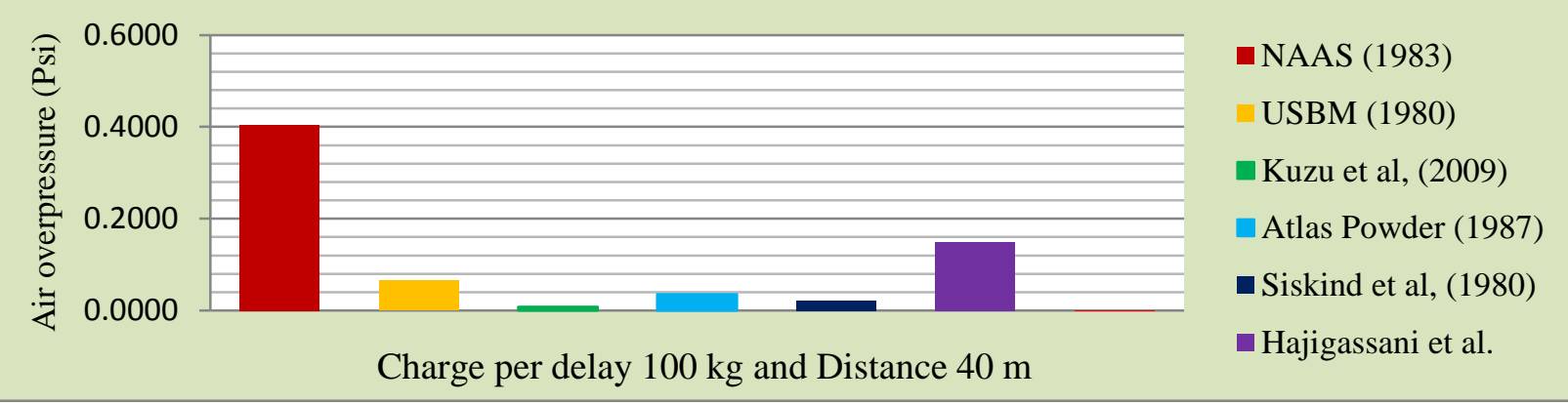

Figure 10. The results of air overpressure for charge per delay $100 \mathrm{~kg}$ and distance $40 \mathrm{~m}$

\section{RESULTS AND DISCUSSION}

In this paper, National Association of Australian State Road Authorities, NAASRA (1983) method is used to predict the air overpressure level for quarry mines. Table 3 to 8 show the results of air overpressure level with the various distance and charge per delay.

Table 3. The results of air overpressure (Psi) with the various distance and charge per delay (A)

\begin{tabular}{|c|c|c|c|c|c|c|c|c|}
\hline \multirow{2}{*}{$\begin{array}{l}\text { Distance } \\
\text { (m) }\end{array}$} & \multicolumn{8}{|c|}{ Charge per delay $(\mathrm{kg})$} \\
\hline & 25 & 50 & 75 & 100 & 125 & 150 & 175 & 200 \\
\hline 20 & 0.511 & 0.642 & 0.734 & 0.807 & 0.869 & 0.923 & 0.971 & 1.015 \\
\hline 30 & 0.341 & 0.428 & 0.490 & 0.538 & 0.579 & 0.615 & 0.647 & 0.677 \\
\hline 40 & 0.255 & 0.321 & 0.367 & 0.404 & 0.435 & 0.462 & 0.486 & 0.507 \\
\hline 50 & 0.204 & 0.257 & 0.294 & 0.323 & 0.348 & 0.369 & 0.388 & 0.406 \\
\hline 60 & 0.170 & 0.214 & 0.245 & 0.269 & 0.290 & 0.308 & 0.324 & 0.338 \\
\hline 70 & 0.146 & 0.184 & 0.210 & 0.231 & 0.248 & 0.264 & 0.277 & 0.290 \\
\hline 80 & 0.128 & 0.161 & 0.184 & 0.202 & 0.217 & 0.231 & 0.243 & 0.254 \\
\hline 90 & 0.114 & 0.143 & 0.163 & 0.179 & 0.193 & 0.205 & 0.216 & 0.226 \\
\hline 100 & 0.102 & 0.128 & 0.147 & 0.161 & 0.174 & 0.185 & 0.194 & 0.203 \\
\hline 110 & 0.093 & 0.117 & 0.134 & 0.147 & 0.158 & 0.168 & 0.177 & 0.185 \\
\hline 120 & 0.085 & 0.107 & 0.122 & 0.135 & 0.145 & 0.154 & 0.162 & 0.169 \\
\hline 130 & 0.079 & 0.099 & 0.113 & 0.124 & 0.134 & 0.142 & 0.149 & 0.156 \\
\hline
\end{tabular}


International Journal of Advances in Scientific Research and Engineering (ijasre), Vol 5 (10), October-2019

\begin{tabular}{|l|l|l|l|l|l|l|l|l|}
\hline 140 & 0.073 & 0.092 & 0.105 & 0.115 & 0.124 & 0.132 & 0.139 & 0.145 \\
\hline 150 & 0.068 & 0.086 & 0.098 & 0.108 & 0.116 & 0.123 & 0.129 & 0.135 \\
\hline 160 & 0.064 & 0.080 & 0.092 & 0.101 & 0.109 & 0.115 & 0.121 & 0.127 \\
\hline 170 & 0.060 & 0.076 & 0.086 & 0.095 & 0.102 & 0.109 & 0.114 & 0.119 \\
\hline 180 & 0.057 & 0.071 & 0.082 & 0.090 & 0.097 & 0.103 & 0.108 & 0.113 \\
\hline 190 & 0.054 & 0.068 & 0.077 & 0.085 & 0.091 & 0.097 & 0.102 & 0.107 \\
\hline 200 & 0.051 & 0.064 & 0.073 & 0.081 & 0.087 & 0.092 & 0.097 & 0.101 \\
\hline
\end{tabular}

Table 4. The results of air overpressure (Psi) with the various distance and charge per delay (B)

\begin{tabular}{|c|c|c|c|c|c|c|c|c|}
\hline \multirow{2}{*}{$\begin{array}{c}\text { Distance } \\
(\mathrm{m})\end{array}$} & \multicolumn{7}{|c|}{ Charge per delay $(\mathrm{kg})$} \\
\hline & 225 & 250 & 275 & 300 & 325 & 350 & 375 & 400 \\
\hline 20 & 1.055 & 1.092 & 1.127 & 1.160 & 1.191 & 1.221 & 1.249 & 1.276 \\
\hline 30 & 0.703 & 0.728 & 0.752 & 0.773 & 0.794 & 0.814 & 0.833 & 0.851 \\
\hline 40 & 0.528 & 0.546 & 0.564 & 0.580 & 0.596 & 0.610 & 0.624 & 0.638 \\
\hline 50 & 0.422 & 0.437 & 0.451 & 0.464 & 0.477 & 0.488 & 0.500 & 0.510 \\
\hline 60 & 0.352 & 0.364 & 0.376 & 0.387 & 0.397 & 0.407 & 0.416 & 0.425 \\
\hline 70 & 0.301 & 0.312 & 0.322 & 0.331 & 0.340 & 0.349 & 0.357 & 0.365 \\
\hline 80 & 0.264 & 0.273 & 0.282 & 0.290 & 0.298 & 0.305 & 0.312 & 0.319 \\
\hline 90 & 0.234 & 0.243 & 0.251 & 0.258 & 0.265 & 0.271 & 0.278 & 0.284 \\
\hline 100 & 0.211 & 0.218 & 0.225 & 0.232 & 0.238 & 0.244 & 0.250 & 0.255 \\
\hline 110 & 0.192 & 0.199 & 0.205 & 0.211 & 0.217 & 0.222 & 0.227 & 0.232 \\
\hline 120 & 0.176 & 0.182 & 0.188 & 0.193 & 0.199 & 0.203 & 0.208 & 0.213 \\
\hline 130 & 0.162 & 0.168 & 0.173 & 0.178 & 0.183 & 0.188 & 0.192 & 0.196 \\
\hline 140 & 0.151 & 0.156 & 0.161 & 0.166 & 0.170 & 0.174 & 0.178 & 0.182 \\
\hline 150 & 0.141 & 0.146 & 0.150 & 0.155 & 0.159 & 0.163 & 0.167 & 0.170 \\
\hline 160 & 0.132 & 0.137 & 0.141 & 0.145 & 0.149 & 0.153 & 0.156 & 0.159 \\
\hline 170 & 0.124 & 0.129 & 0.133 & 0.136 & 0.140 & 0.144 & 0.147 & 0.150 \\
\hline 180 & 0.117 & 0.121 & 0.125 & 0.129 & 0.132 & 0.136 & 0.139 & 0.142 \\
\hline 190 & 0.111 & 0.115 & 0.119 & 0.122 & 0.125 & 0.129 & 0.131 & 0.134 \\
\hline 200 & 0.106 & 0.109 & 0.113 & 0.116 & 0.119 & 0.122 & 0.125 & 0.128 \\
\hline
\end{tabular}

Table 5. The results of air overpressure (Psi) with the various distance and charge per delay (C)

\begin{tabular}{|c|c|c|c|c|c|c|c|c|}
\hline \multirow{2}{*}{$\begin{array}{c}\text { Distance } \\
(\mathrm{m})\end{array}$} & \multicolumn{9}{|c|}{ Charge per delay $(\mathrm{kg})$} \\
\hline & 25 & 50 & 75 & 100 & 125 & 150 & 175 & 200 \\
\hline 210 & 0.049 & 0.061 & 0.070 & 0.077 & 0.083 & 0.088 & 0.092 & 0.097 \\
\hline 220 & 0.046 & 0.058 & 0.067 & 0.073 & 0.079 & 0.084 & 0.088 & 0.092 \\
\hline 230 & 0.044 & 0.056 & 0.064 & 0.070 & 0.076 & 0.080 & 0.084 & 0.088 \\
\hline 240 & 0.043 & 0.054 & 0.061 & 0.067 & 0.072 & 0.077 & 0.081 & 0.085 \\
\hline 250 & 0.041 & 0.051 & 0.059 & 0.065 & 0.070 & 0.074 & 0.078 & 0.081 \\
\hline 260 & 0.039 & 0.049 & 0.056 & 0.062 & 0.067 & 0.071 & 0.075 & 0.078 \\
\hline 270 & 0.038 & 0.048 & 0.054 & 0.060 & 0.064 & 0.068 & 0.072 & 0.075 \\
\hline 280 & 0.036 & 0.046 & 0.052 & 0.058 & 0.062 & 0.066 & 0.069 & 0.072 \\
\hline 290 & 0.035 & 0.044 & 0.051 & 0.056 & 0.060 & 0.064 & 0.067 & 0.070 \\
\hline 300 & 0.034 & 0.043 & 0.049 & 0.054 & 0.058 & 0.062 & 0.065 & 0.068 \\
\hline 310 & 0.033 & 0.041 & 0.047 & 0.052 & 0.056 & 0.060 & 0.063 & 0.065 \\
\hline 320 & 0.032 & 0.040 & 0.046 & 0.050 & 0.054 & 0.058 & 0.061 & 0.063 \\
\hline 330 & 0.031 & 0.039 & 0.045 & 0.049 & 0.053 & 0.056 & 0.059 & 0.062 \\
\hline 340 & 0.030 & 0.038 & 0.043 & 0.047 & 0.051 & 0.054 & 0.057 & 0.060 \\
\hline 350 & 0.029 & 0.037 & 0.042 & 0.046 & 0.050 & 0.053 & 0.055 & 0.058 \\
\hline 360 & 0.028 & 0.036 & 0.041 & 0.045 & 0.048 & 0.051 & 0.054 & 0.056 \\
\hline 370 & 0.028 & 0.035 & 0.040 & 0.044 & 0.047 & 0.050 & 0.052 & 0.055 \\
\hline 380 & 0.027 & 0.034 & 0.039 & 0.042 & 0.046 & 0.049 & 0.051 & 0.053 \\
\hline 390 & 0.026 & 0.033 & 0.038 & 0.041 & 0.045 & 0.047 & 0.050 & 0.052 \\
\hline 400 & 0.026 & 0.032 & 0.037 & 0.040 & 0.043 & 0.046 & 0.049 & 0.051 \\
\hline
\end{tabular}

Table 6. The results of air overpressure (Psi) with the various distance and charge per delay (D)

\begin{tabular}{|c|c|c|c|c|c|c|c|c|}
\hline \multirow{2}{*}{$\begin{array}{c}\text { Distance } \\
(\mathrm{m})\end{array}$} & \multicolumn{7}{|c|}{ Charge per delay (kg) } \\
\cline { 2 - 8 } & 225 & 250 & 275 & 300 & 325 & 350 & 375 & 400 \\
\hline 210 & 0.100 & 0.104 & 0.107 & 0.110 & 0.113 & 0.116 & 0.119 & 0.122 \\
\hline 220 & 0.096 & 0.099 & 0.102 & 0.105 & 0.108 & 0.111 & 0.114 & 0.116 \\
\hline
\end{tabular}


International Journal of Advances in Scientific Research and Engineering (ijasre), Vol 5 (10), October-2019

\begin{tabular}{|l|l|l|l|l|l|l|l|l|}
\hline 230 & 0.092 & 0.095 & 0.098 & 0.101 & 0.104 & 0.106 & 0.109 & 0.111 \\
\hline 240 & 0.088 & 0.091 & 0.094 & 0.097 & 0.099 & 0.102 & 0.104 & 0.106 \\
\hline 250 & 0.084 & 0.087 & 0.090 & 0.093 & 0.095 & 0.098 & 0.100 & 0.102 \\
\hline 260 & 0.081 & 0.084 & 0.087 & 0.089 & 0.092 & 0.094 & 0.096 & 0.098 \\
\hline 270 & 0.078 & 0.081 & 0.084 & 0.086 & 0.088 & 0.090 & 0.093 & 0.095 \\
\hline 280 & 0.075 & 0.078 & 0.081 & 0.083 & 0.085 & 0.087 & 0.089 & 0.091 \\
\hline 290 & 0.073 & 0.075 & 0.078 & 0.080 & 0.082 & 0.084 & 0.086 & 0.088 \\
\hline 300 & 0.070 & 0.073 & 0.075 & 0.077 & 0.079 & 0.081 & 0.083 & 0.085 \\
\hline 310 & 0.068 & 0.070 & 0.073 & 0.075 & 0.077 & 0.079 & 0.081 & 0.082 \\
\hline 320 & 0.066 & 0.068 & 0.070 & 0.073 & 0.074 & 0.076 & 0.078 & 0.080 \\
\hline 330 & 0.064 & 0.066 & 0.068 & 0.070 & 0.072 & 0.074 & 0.076 & 0.077 \\
\hline 340 & 0.062 & 0.064 & 0.066 & 0.068 & 0.070 & 0.072 & 0.073 & 0.075 \\
\hline 350 & 0.060 & 0.062 & 0.064 & 0.066 & 0.068 & 0.070 & 0.071 & 0.073 \\
\hline 360 & 0.059 & 0.061 & 0.063 & 0.064 & 0.066 & 0.068 & 0.069 & 0.071 \\
\hline 370 & 0.057 & 0.059 & 0.061 & 0.063 & 0.064 & 0.066 & 0.068 & 0.069 \\
\hline 380 & 0.056 & 0.057 & 0.059 & 0.061 & 0.063 & 0.064 & 0.066 & 0.067 \\
\hline 390 & 0.054 & 0.056 & 0.058 & 0.059 & 0.061 & 0.063 & 0.064 & 0.065 \\
\hline 400 & 0.053 & 0.055 & 0.056 & 0.058 & 0.060 & 0.061 & 0.062 & 0.064 \\
\hline
\end{tabular}

Table 7. The results of air overpressure (Psi) with the various distance and charge per delay (E)

\begin{tabular}{|c|c|c|c|c|c|c|c|c|}
\hline \multirow{2}{*}{$\begin{array}{c}\text { Distance } \\
(\mathrm{m})\end{array}$} & \multicolumn{9}{|c|}{ Charge per delay $(\mathrm{kg})$} \\
\cline { 2 - 8 } & 25 & 50 & 75 & 100 & 125 & 150 & 175 & 200 \\
\hline 410 & 0.025 & 0.031 & 0.036 & 0.039 & 0.042 & 0.045 & 0.047 & 0.050 \\
\hline 420 & 0.024 & 0.031 & 0.035 & 0.038 & 0.041 & 0.044 & 0.046 & 0.048 \\
\hline 430 & 0.024 & 0.030 & 0.034 & 0.038 & 0.040 & 0.043 & 0.045 & 0.047 \\
\hline 440 & 0.023 & 0.029 & 0.033 & 0.037 & 0.040 & 0.042 & 0.044 & 0.046 \\
\hline 450 & 0.023 & 0.029 & 0.033 & 0.036 & 0.039 & 0.041 & 0.043 & 0.045 \\
\hline 460 & 0.022 & 0.028 & 0.032 & 0.035 & 0.038 & 0.040 & 0.042 & 0.044 \\
\hline 470 & 0.022 & 0.027 & 0.031 & 0.034 & 0.037 & 0.039 & 0.041 & 0.043 \\
\hline 480 & 0.021 & 0.027 & 0.031 & 0.034 & 0.036 & 0.038 & 0.040 & 0.042 \\
\hline 490 & 0.021 & 0.026 & 0.030 & 0.033 & 0.035 & 0.038 & 0.040 & 0.041 \\
\hline 500 & 0.020 & 0.026 & 0.029 & 0.032 & 0.035 & 0.037 & 0.039 & 0.041 \\
\hline 510 & 0.020 & 0.025 & 0.029 & 0.032 & 0.034 & 0.036 & 0.038 & 0.040 \\
\hline 520 & 0.020 & 0.025 & 0.028 & 0.031 & 0.033 & 0.036 & 0.037 & 0.039 \\
\hline 530 & 0.019 & 0.024 & 0.028 & 0.030 & 0.033 & 0.035 & 0.037 & 0.038 \\
\hline 540 & 0.019 & 0.024 & 0.027 & 0.030 & 0.032 & 0.034 & 0.036 & 0.038 \\
\hline 550 & 0.019 & 0.023 & 0.027 & 0.029 & 0.032 & 0.034 & 0.035 & 0.037 \\
\hline 560 & 0.018 & 0.023 & 0.026 & 0.029 & 0.031 & 0.033 & 0.035 & 0.036 \\
\hline 570 & 0.018 & 0.023 & 0.026 & 0.028 & 0.030 & 0.032 & 0.034 & 0.036 \\
\hline 580 & 0.018 & 0.022 & 0.025 & 0.028 & 0.030 & 0.032 & 0.033 & 0.035 \\
\hline 590 & 0.017 & 0.022 & 0.025 & 0.027 & 0.029 & 0.031 & 0.033 & 0.034 \\
\hline 600 & 0.017 & 0.021 & 0.024 & 0.027 & 0.029 & 0.031 & 0.032 & 0.034 \\
\hline
\end{tabular}

Table 8. The results of air overpressure (Psi) with the various distance and charge per delay (F)

\begin{tabular}{|c|c|c|c|c|c|c|c|c|}
\hline \multirow{2}{*}{$\begin{array}{c}\text { Distance } \\
(\mathrm{m})\end{array}$} & \multicolumn{7}{|c|}{ Charge per delay $(\mathrm{kg})$} \\
\hline & 225 & 250 & 275 & 300 & 325 & 350 & 375 & 400 \\
\hline 410 & 0.051 & 0.053 & 0.055 & 0.057 & 0.058 & 0.060 & 0.061 & 0.062 \\
\hline 420 & 0.050 & 0.052 & 0.054 & 0.055 & 0.057 & 0.058 & 0.059 & 0.061 \\
\hline 430 & 0.049 & 0.051 & 0.052 & 0.054 & 0.055 & 0.057 & 0.058 & 0.059 \\
\hline 440 & 0.048 & 0.050 & 0.051 & 0.053 & 0.054 & 0.055 & 0.057 & 0.058 \\
\hline 450 & 0.047 & 0.049 & 0.050 & 0.052 & 0.053 & 0.054 & 0.056 & 0.057 \\
\hline 460 & 0.046 & 0.047 & 0.049 & 0.050 & 0.052 & 0.053 & 0.054 & 0.055 \\
\hline 470 & 0.045 & 0.046 & 0.048 & 0.049 & 0.051 & 0.052 & 0.053 & 0.054 \\
\hline 480 & 0.044 & 0.046 & 0.047 & 0.048 & 0.050 & 0.051 & 0.052 & 0.053 \\
\hline 490 & 0.043 & 0.045 & 0.046 & 0.047 & 0.049 & 0.050 & 0.051 & 0.052 \\
\hline 500 & 0.042 & 0.044 & 0.045 & 0.046 & 0.048 & 0.049 & 0.050 & 0.051 \\
\hline 510 & 0.041 & 0.043 & 0.044 & 0.045 & 0.047 & 0.048 & 0.049 & 0.050 \\
\hline 520 & 0.041 & 0.042 & 0.043 & 0.045 & 0.046 & 0.047 & 0.048 & 0.049 \\
\hline 530 & 0.040 & 0.041 & 0.043 & 0.044 & 0.045 & 0.046 & 0.047 & 0.048 \\
\hline 540 & 0.039 & 0.040 & 0.042 & 0.043 & 0.044 & 0.045 & 0.046 & 0.047 \\
\hline
\end{tabular}


International Journal of Advances in Scientific Research and Engineering (ijasre), Vol 5 (10), October-2019

\begin{tabular}{|l|l|l|l|l|l|l|l|l|}
\hline 550 & 0.038 & 0.040 & 0.041 & 0.042 & 0.043 & 0.044 & 0.045 & 0.046 \\
\hline 560 & 0.038 & 0.039 & 0.040 & 0.041 & 0.043 & 0.044 & 0.045 & 0.046 \\
\hline 570 & 0.037 & 0.038 & 0.040 & 0.041 & 0.042 & 0.043 & 0.044 & 0.045 \\
\hline 580 & 0.036 & 0.038 & 0.039 & 0.040 & 0.041 & 0.042 & 0.043 & 0.044 \\
\hline 590 & 0.036 & 0.037 & 0.038 & 0.039 & 0.040 & 0.041 & 0.042 & 0.043 \\
\hline 600 & 0.035 & 0.036 & 0.038 & 0.039 & 0.040 & 0.041 & 0.042 & 0.043 \\
\hline
\end{tabular}

To obtain the values of air overpressure, it is necessary to find out values of site constants $\mathrm{H}$ and B. Site constants are main factors for prediction of air overpressure level due to blasting. Site constants can be determined by regression analysis. In this study, site constants are used directly from the book values. Generally high overpressure levels are caused due to inadequate stemming, mud or weak seam venting, inadequate burden confinement, poor blasting timing, focusing by wind or temperature inversions, uncovered detonation cord and overloading.

\section{CONCLUSION}

Blast-induced airblast or overpressure is one of the negative effects of blasting operations. The resulting noise usually generates a lot of uneasiness and irritation to neighbors giving rise to complaints. Blast-induced airblast can be minimized by properly designing and implementing blasts. The main cause of high frequency airblast tends to be holes blowing out, either by ejecting the stemming or by venting through mud seams. The expected air blast levels from blasting operations were calculated and considered in relation to the surrounding structures and installations.

To limit the air blast safe level, based on work carried out by Siskind et.al. (1980), monitored air blast amplitudes up to $135 \mathrm{~dB}$ are safe for structures, provided the monitoring instrument is sensitive to low frequencies (down to $1 \mathrm{~Hz}$ ). [5] This paper focuses on the prediction of air overpressure level that is used for Myanmar quarry mines.

\section{REFERENCES}

[1] J. Ratcliff., Ed Sheehan., and K. Carte, "Predictability of airblast at surface coal mines in west virginia," Department of Environmental Protection Office of Explosives and Blasting,West Virginia, pp -11, 2011.

[2] M. Hajihassani, D. J. Armaghani, and M. Monjezi, "Blast-induce air and ground vibration prediction: a particle swarm optimization-based artificial neural network approach," Environmental Earth Sciences, DOI 10.1007/s12665-015-4274-1.March 2015.

[3] W. L. Bender, "Understanding blast vibration and airblast, their causes, and their damage potential," at the Spring 2006 and Fall 2007 workshops of the Golden West Chapter of the International Society of Explosives Engineers. pp-6, 2017.

[4] R. Agne," Rock blasting terms and symbols," Division of Mining Engineering, Luled University of Technology, Sweden. 1998.

[5] J.D. Zeeman, “Ground vibration and air blast Study by blast management \& consulting," Social and Environmental Impact Assessment: Ground Vibration and Air Blast Study for Rio Tinto, Rössing Uranium Mine Expansion Project, Namibia. Dated 31 January 2009.

[6] R. N. Tiile, "Artificial neural network approach to predict blast-induced ground vibration, airblast and rock fragmentation," Master of Science In Mining Engineering, Missouri University of Science and Technology. pp -18, 2016.

[7] A. Parida, " Evaluation of blasting efficiency in surface mines," Master of Technology in Mining Engineering, National Institute of Technology Rourkela. Pp 22- 23, December, 2016.

\section{AUTHORS}

First Author - Zaw Moon Aung, Demonstrator, Mandalay Technological University and zawmoonaung2017@ gmail.com Second Author - Myo Min Lwin, Professor, Mandalay Technological University and sayarmyo02@ gmail.com 